\title{
MOMENTO OPTIMO DE TRASPLANTE, BAJ O DIFERENTES MODALIDADES EN PLANTONES DE CAMU-CAMU (Myrciaria dubia Mc Vaugh)
}

Armando Vásquez *

Martha Gastelo**

\section{RESUMEN}

El presente experimento, el segundo en su serie, se llevó a cabo en las instalaciones del fundo "Zungaro Cocha" de propiedad de la Facultad de Agronomía de la Universidad Nacional de la Amazonía Peruana, a 25 $\mathrm{km}$ de la ciudad de lquitos, cuyo objetivo fue la de determinar el momento óptimo de trasplante bajo diferentes modalidades; para tal efecto se sembraron semillas de camu-camu procedentes del lago "Supay" (río Ucayali); cuando las plántulas tuvieron entre 10 y $12 \mathrm{~cm}$ de altura en el almácigo se efectuó el repique y con ella el primer trasplante.

Se determinó que el mejor momento para el trasplante, corresponde a los 30 días después del repique y bajo la modalidad con pan de tierra, pudiendo ser efectivo también a los 15 y 45 días bajo la misma modalidad.

\section{SUMMARY}

For the present work the second experiment in "Zungaro Cocha" farm instalación Agronomic Faculty (U.N.A.P.), localated at $25 \mathrm{~km}$ from Iquitos city. This papel show the best time to transplant under diferent conditions. It was showed camu-camu seeds from "Supay" lake (Ucayali river); when the small plants were $10-12 \mathrm{~cm}$ height w orw transplanted.

It $w$ as found that the best time to replant was 30 days after the pealing, using earth bread, too it? possible betwen 15 to 45 days.

Palabras claves: Trasplante, Modalidad de trasplante, Myrciaria dubia, camu camu.

Ingo Ms. Docente, Investigador de la UNAP, Responsable Proyecto Estudio Integral del camu-camu.

** Ing@ Agrónomo Co-investigador Proyecto Estudio Integral del camu-camu. 


\section{INTRODUCCION}

El camu-camu (Myrciaria dubia Mc. Vaugh), es una especie frutal nativa de la Amazonía peruana de la familia de las Myrtaceas y notables por su elevada concentración de ácido ascórbico (2888 mg en $100 \mathrm{gr}$ de pulpa) Roca (1965).

El presente trabajo, el segundo en su serie, informa sobre el manejo de esta especie a nivel de vivero (el primero fue a nivel de almácigo) y describe las condiciones necesarias para transplantar el camu-camu en terreno definitivo en el menor tiempo posible y las condiciones para dicho transplante. La importancia de este experimento radica en el hecho de que cuanto mayor tiempo tengamos a los plantones en vivero, tanto menor será su desarrollo en terreno definitivo, y por el contrario cuanto más rápido hagamos el transplante (en su momento óptimo), tanto mayor será su desarrollo Calzada (1985).

Ante esta situación el Proyecto Estudio Integral del Camu-camu de la Facultad de Agronomía U.N.A.P. ejecutó el presente estudio, cuyo objetivo fue determinar el momento óptimo (tiempo), bajo diversas modalidades de transplante en plantones de camu-camu.

\section{ANTECEDENTES}

Gutiérrez (1969), informa que el camu-camu debe ser llevado a terreno definitivo (trasplante) a los 270 días (9 meses), con una altura promedio de $40 \mathrm{~cm}$ y aproximadamente con 40 hojas.

Calzada (1985), reporta que cuanto más rápido trasplantemos al camu-camu tanto mayor será su desarrollo, recomendando llevarlo a terreno definitivo a lo más a tres meses después de haberlo sembrado en almácigo.

Pinedo M. y Utia (1992), informan en un experimento de trasplante efectuado en camu-camu, arazá y pijuayo, con pan de tierra y a raíz desnuda y a diferentes edades de los plantones $(5,7,9$ y 11 meses), concluyen que en camu-camu específicamente, no se observó influencia significativa tanto en supervivencia como en altura, recomendando efectuar el trasplante a raíz desnuda, luego de 5 a 9 meses de almacigado. 


\section{MATERIALES Y METODOS}

El presente estudio fue conducido durante los años 1993, 1994, en el fundo Zungaro Cocha de propiedad de la Facultad de Agronomía (UNAP), correspondiente a un suelo de terraza alta tipo ultisol y con precipitaciones pluviales que oscilan entre 2000 a $4000 \mathrm{~mm}$ anuales y una temperatura promedio de $28^{\circ} \mathrm{C}$.

Las semillas en estudio fueron colectadas del lago Supay (río Ucayali), muy cerca a la localidad de J enaro Herrera, donde existe una población natural de camu-camu de aproximadamente 60 ha Peters y Vásquez, (1987); estas semillas fueron tratadas (quitar su musílago y lavar) y seleccionadas, para luego almacigarlas en camas ya preparadas para este fin, fueron sembradas a un distanciamiento de $3 \mathrm{~cm}$ entre semillas y $10 \mathrm{~cm}$ entre líneas, a una profundidad de $3 \mathrm{~cm}$ esta operación se efectuo el 8 de diciembre de 1992, haciendo un total de 1000 semillas aproximadamente por cama de $8 \times 1 \mathrm{~m}$.

La germinación se inició a los 29 días con un porcentaje de germinación entre 95 y 100 \%, luego el repique se efectuó cuando las plántulas tuvieron entre 10 y $12 \mathrm{~cm}$ de altura, esta labor se realizó el 21 de enero de 1993 y con ella se inició los tratamientos en estudio que resultó de la combinación de los dos factores que son:

Factor A : Momento de trasplante (tiempo)

A0 : $\quad$ Trasplante al repique.

A1 : $\quad$ Trasplante a 15 días después del repique.

A2 : $\quad$ Trasplante a 30 días después del repique.

A3 : $\quad$ Trasplante a 45 días después del repique.

Factor B : Modalidad de transplante *
B1 : $\quad$ Con pan de tierra.
B2 : $\quad$ Raíz desnuda.
B3 : $\quad$ Deshojado.
B4 : $\quad$ Pseudoestaca.

* Los dibujos de cada modalidad se encuentran en el anexo 2. 
De la combinación de estos niveles cuatro de A y cuatro de B nos da el factorial $4 \times 4$, que representan 16 tratamientos en estudio; así mismo el ANVA tuvo las siguientes fuentes de variabilidad:

\begin{tabular}{||l|l||}
\hline Fuente & $\mathrm{GL}$ \\
\hline Reproducciones & $\mathrm{r}-1=2$ \\
\hline A : Trasplante & $\mathrm{a}-1=3$ \\
\hline B : Modalidad & $\mathrm{b}-1=3$ \\
\hline A x B & $(\mathrm{a}-1)(\mathrm{b}-1)=9$ \\
\hline Error & $(\mathrm{r}-1)(\mathrm{ab}-1)=16$ \\
\hline Total & $\mathrm{abr}-1=47$ \\
\hline
\end{tabular}

El primer trasplante se efectuó el 21 de enero de 1993, el segundo el 5 de febrero de 1993, el tercero el 20 de febrero de 1993 y el último el 7 de marzo de 1993 con 60 plántulas por tratamiento y un total de 240 por repetición; en cada tratamiento se tomaron los siguientes datos : porcentaje de prendimiento, altura de las plantas, número de hojas y diametro del tallo.

Sembradas las plántulas en terreno definitivo se efectuó la revisión cada 10 días, se contabilizaron el número de plántulas muertas por cada tratamiento y repetición y por diferencia se secaba el porcentaje de prendimiento; luego para el análisis estadístico fueron transformados a la función arco sen X para su tabulación; posteriormente se trabajo con la variable altura que también fue incluido en el análisis estadístico debido a la notable diferencia de altura de las plantas en todo los tratamientos al cuarto mes que estuvieron en terreno definitivo.

\section{RESULTADOS}

Se analizaron las dos variables más importantes como son porcentaje de prendimiento y altura de las plantas, que se muestran en los cuadros siguientes. 
A nalisis variable porcentaje de prendimiento.

\section{Cuadro 1}

Análisis de varianza, variable porcentaje de prendimiento (\%) ANVA

\begin{tabular}{|c|c|r|r|r|r|r||}
\hline Fuente & \multicolumn{1}{|c|}{ G.L } & S.C. & C.M. & F.C. & F 0.01 & Significación \\
\hline Bloques & \multicolumn{1}{|c|}{$3-1=2$} & 934.33 & 467.16 & 6.14 & 3.32 & $*$ \\
$\begin{array}{c}\text { Tratamientos } \\
\text { (combinado) }\end{array}$ & $16-1=15$ & 4313.69 & & & \\
A & $4-1=3$ & 317.62 & 105.87 & 1.39 & 2.92 & n.s. \\
B & $4-1=3$ & 3103.11 & 1034.37 & 13.6 & 2.92 & $*$ \\
A x B & $(4-1)(4-1)=9$ & 892.96 & 99.21 & 1.3 & 2.25 & n.s. \\
Error & $(\mathrm{r}-1)(\mathrm{ab}-1)=30$ & 2281.03 & 76.03 & & & \\
\hline Total & & 11842.74 & & & & \\
\hline
\end{tabular}

$$
\begin{aligned}
& \text { C.V. }=\frac{\mathrm{V} 76.03 \times 100}{221.40} \\
& \mathrm{C.V} .=3.39
\end{aligned}
$$

* Diferencia significativa en repeticiones y en modalidad de trasplante. Dada la significancia en $B$, se procedió a realizar la prueba de Duncan. 


\section{Cuadro 2.}

Tabla de significación para factor B (modalidad de trasplante) y significativo al ANVA; variable porcentaje de prendimiento.

\begin{tabular}{|c|c|c|}
\hline $\begin{array}{l}\text { Medias por tratamiento Factor B } \\
\text { Clave Descripción }\end{array}$ & $X$ & $\begin{array}{c}\text { Duncan } \\
\mathrm{a}=0.05\end{array}$ \\
\hline $\begin{array}{ll}\text { B1 } & \text { Pan de tierra }\end{array}$ & 86.66 & $a$ \\
\hline Deshojado & 73.16 & a \\
\hline Raíz desnuda & 69.03 & $b$ \\
\hline Pseudoestaca & 65.56 & $b$ \\
\hline
\end{tabular}

El cuadro nos muestra hasta dos grupos homogéneos, obteniendo el mayor promedio de porcentaje de prendimiento el transplantado en forma deshojado; ambos forman un grupo homogéneo, la forma de pseudoestaca resultó con el promedio más bajo de prendimiento (65.56)

\section{Cuadro 3}

Tabla de significación para factor A (momento óptimo de transplante) y significativo al ANVA; variable porcentaje de prendimiento.

\begin{tabular}{|c|c|c|}
\hline $\begin{array}{l}\text { Medias por tratamiento Factor A } \\
\text { Clave Descripción }\end{array}$ & $X$ & $\begin{array}{l}\text { Duncan } \\
a=0.05\end{array}$ \\
\hline A3a $\quad 45$ días & 77.83 & $a$ \\
\hline al repique & 74.20 & a \\
\hline a 30 días & 71.35 & a \\
\hline a 15 días & 71.14 & a \\
\hline
\end{tabular}

El cuadro nos muestra un solo grupo homogéneo, ligeramente el transplante a 45 días es el mejor, sin embargo no resulta significativo. 


\section{Cuadro 4}

Medias de Tratamiento ** Variable porcentaje de Prendimiento

\begin{tabular}{||c|c|c|c|c|c||}
\hline $\begin{array}{c}\text { Momento } \\
\text { Optimo }\end{array}$ & \multicolumn{3}{|c|}{ Modalidadde trasplante } & & \multirow{2}{*}{$\mathrm{X}$} \\
\cline { 2 - 5 } & Pan de Tierra & Raíz desnuda & Deshojado & Pseudoestaca & \\
\hline al repique & $81.38(4.30)^{*}$ & $81.14(8.85)$ & $71.88(2.86)$ & $62.46(2.40)$ & $74.22(4.21)$ \\
a 15 días & $85.69(4.30)$ & $63.86(4.21)$ & $68.02(4.64)$ & $67.02(3.85)$ & $71.15(4.92)$ \\
a 30 días & $90.00(0.0)$ & $61.14(1.74)$ & $71.38(5.69)$ & $62.88(13.58)$ & $71.35(6.60)$ \\
a 45 días & $90.00(0.0)$ & $\begin{array}{c}70.00 \\
(10.00)\end{array}$ & $81.38(4.30)$ & $69.97(1.38)$ & $77.84(4.63)$ \\
\hline$x$ & $86.77(2.06)$ & $69.04(4.44)$ & $73.17(1.97)$ & $65.58(1.67)$ & 73.64 \\
\hline
\end{tabular}

Los números entre paréntesis corresponden a la desviación standard de las medias.

Datos obtenidos del cuadro 1 del anexo. 
Grafico 1: Representación gráfica de las medias de tratamiento (efectos principales), datos tomados del cuadro 4, variable porcentaje de prendimiento.

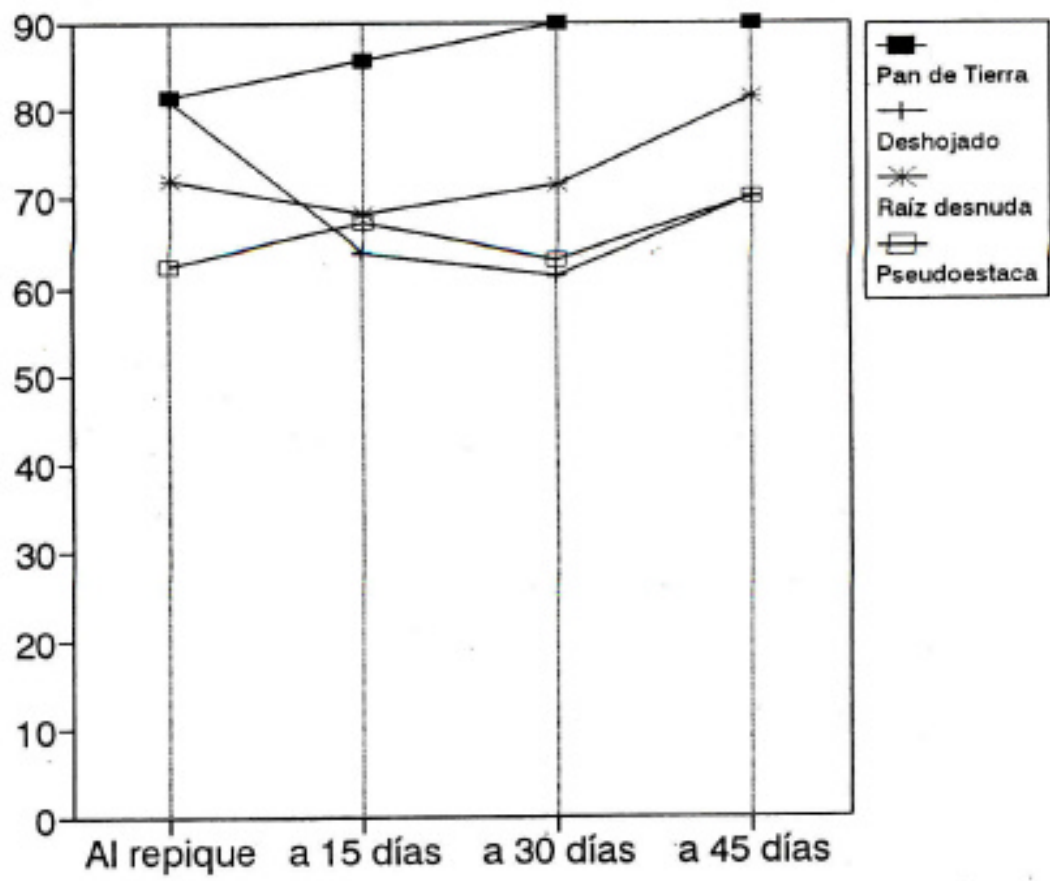


A nálisis de la variable altura de plántulas (datos tomados al 4to mes del trasplante)

\section{Cuadro 5}

Análisis de varianza: variable altura de plántulas $(\mathrm{cm})$ ANVA

\begin{tabular}{|c|c|c|c|c|c|c|}
\hline Fuente & G.L & S.C. & C.M. & F.C. & F 0.01 & Significación \\
\hline $\begin{array}{c}\text { Bloques } \\
\text { Tratamientos } \\
\text { (combinado) }\end{array}$ & $\begin{array}{l}3-1=2 \\
16-1=15\end{array}$ & $\begin{array}{l}40.63 \\
71.91\end{array}$ & 20.31 & 1.329 & 3.32 & n.s. \\
\hline A & $4-1=3$ & 605.56 & 201.85 & 13.21 & 2.92 & * \\
\hline B & $4-1=3$ & 5856.41 & 1952.13 & 127.75 & 2.92 & $* *$ \\
\hline$A \times B$ & $(4-1)(4-1)=9$ & 729.39 & 81.04 & 5.3 & 2.21 & * \\
\hline Error & $(r-1)(a b-1)=30$ & 458.63 & 15.28 & & & \\
\hline Total & & 7762.53 & & & & \\
\hline
\end{tabular}

$$
\begin{aligned}
& \text { C.V. }=\frac{v 81.04 \times 100}{77.33} \\
& \text { C.V. }=12.27
\end{aligned}
$$

* Diferencia significativa en momento optimo de trasplante, (A), y en la interacción A $x$ B dada la significancia en $B$, se procedió a realizar la prueba de Duncan. 


\section{Cuadro 6.}

Tabla de significación, para factor A (momento óptimo) y significativo al ANVA; variable altura de plantas al cuarto mes.

\begin{tabular}{|c|c|c|c|}
\hline \multicolumn{2}{|c|}{$\begin{array}{c}\text { Promedios por tratamiento } \\
\text { Factor A }\end{array}$} & \multirow[t]{2}{*}{$X$} & \multirow[t]{2}{*}{$\begin{array}{l}\text { Duncan } \\
\mathrm{a}=0.05\end{array}$} \\
\hline & Descripción & & \\
\hline A2a & a 30 días & 29.30 & a \\
\hline A3 & a 45 días & 25.72 & a \\
\hline A 1 & a 15 días & 23.12 & a \\
\hline AO & al repique & 19.60 & $b$ \\
\hline
\end{tabular}

El cuadro nos muestra hasta dos grupos homogéneos, indicando que el mejor momento para trasplantar corresponde a los 30 días después del repique (29.30), sin embargo es posible trasplantar también a los 45 y 15 dias respectivamente.

\section{Cuadro 7.}

Tabla de significación, para factor B (modalidad de trasplante) y significativo al ANVA; variable altura de plantas al cuarto mes.

\begin{tabular}{||cc|c|c||}
\hline \hline \multicolumn{2}{|c|}{$\begin{array}{c}\text { Promedios por tratamiento } \\
\text { Factor B } \\
\end{array}$} & $\mathrm{h} \mathrm{X}$ & $\begin{array}{c}\text { Duncan } \\
\mathrm{a}=0.05\end{array}$ \\
\hline B1 & Pescripción & & $\mathrm{a}$ \\
B2 & Pan de tierra & 43.13 & $\mathrm{~b}$ \\
B3 & Raíz desnuda & 20.19 & $\mathrm{~b}$ \\
B4 & Deshojado & 19.97 & $\mathrm{~b}$ \\
\hline
\end{tabular}

En este cuadro se observa tambien dos grupos homogéneos, resultando el mejor tratamiento y con altura significativa al resto de tratamientos el trasplante con Pan de tierra (B1) con $43.13 \mathrm{~cm}$ em promedio al cuarto mes, la Pseudoestaca resulto con el más bajo promedio $14.44 \mathrm{~cm}$. 


\section{Cuadro 8}

\section{Medias de Tratamiento: Variable de Prendimiento}

\begin{tabular}{||c|c|c|c|c|c||}
\hline $\begin{array}{c}\text { Momento } \\
\text { Optimo }\end{array}$ & & Modalidad & de trasplante & & \multirow{2}{*}{$\mathrm{X}$} \\
\cline { 2 - 5 } & $\begin{array}{c}\text { Pan de Tierra } \\
(\mathrm{b})\end{array}$ & $\begin{array}{c}\text { Raíz desnuda } \\
(\mathrm{b} 2)\end{array}$ & $\begin{array}{c}\text { Deshojado } \\
(\mathrm{b} 3)\end{array}$ & $\begin{array}{c}\text { Pseudoestac } \\
\text { a (b4) }\end{array}$ & \\
\hline al repique & $31.15(3.02) *$ & $19.82(4.62)$ & $15.37(1.66)$ & $12.09(0.47)$ & $19.61(4.30)$ \\
a 15 días & $37.71(0.73)$ & $21.56(0.99)$ & $20.70(1.12)$ & $12.52(1.20)$ & $23.12(6.08)$ \\
a 30 días & $57.10(3.15)$ & $18.35(1.80)$ & $23.36(2.30)$ & $18.42(3.20)$ & $29.31(10.78)$ \\
a 45 días & $46.64(2.38)$ & $21.05(1.13)$ & $20.48(0.37)$ & $14.74(2.72)$ & $25.73(8.21)$ \\
\hline$X$ & $43.15(6.50)$ & $20.20(0.82)$ & $19.98(1.97)$ & $14.44(1.67)$ & 24.44 \\
\hline
\end{tabular}

* Los números entre paréntesis corresponden a la desviación standard de las medias. 
Gráfico 2. : Representación gráfica de los efectos principales:

Variable altura de plántulas datos provenientes del cuadro 8. (A dentro de B).

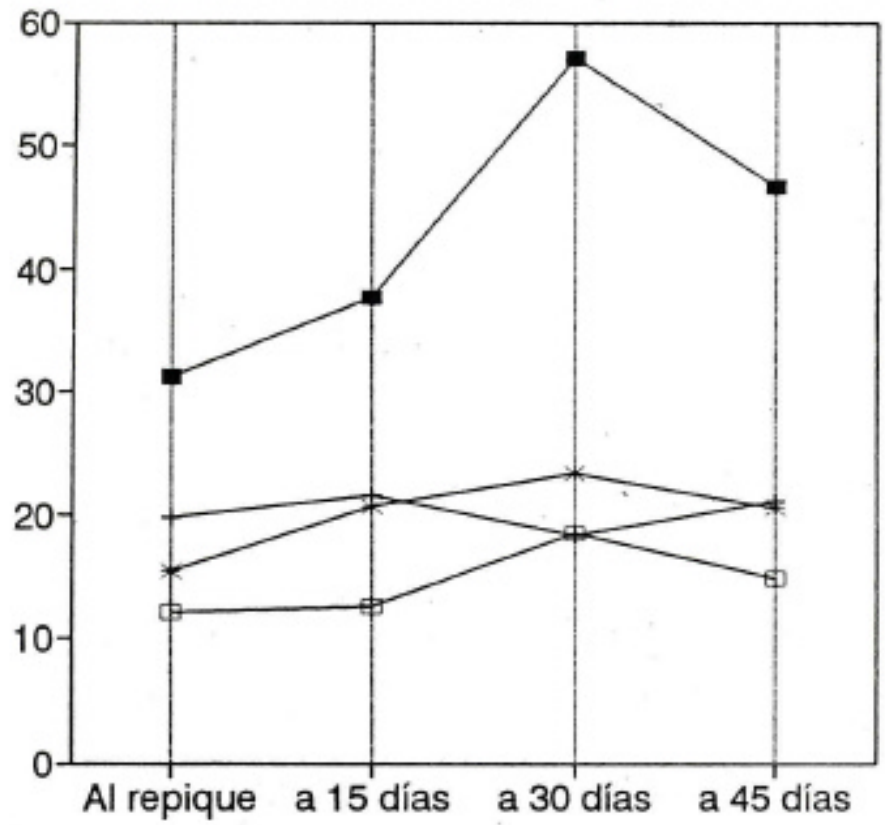

Pan de Tierra

$+$

Deshojado

Aaíz desnuda

E

Pseudoestaca

Al repique a 15 días a 30 dias a 45 dias 
Exámen de la interacción A x B.

\section{Cuadro 9.}

\section{Totales de Tratamiento Interacción A x B}

\begin{tabular}{|c|c|c|c|c|c|c|}
\hline \multirow[t]{2}{*}{ Factor } & \multicolumn{5}{|c|}{$A=M$ omento Optimo } & \multirow[b]{2}{*}{ Totales } \\
\hline & Nivel & $\begin{array}{c}\mathrm{a} 0 \\
\text { al repique }\end{array}$ & \begin{tabular}{|c|} 
a1 \\
a 15 días
\end{tabular} & $\begin{array}{c}\text { a2 } \\
\text { a } 30 \text { días }\end{array}$ & $\begin{array}{c}\text { a3 } \\
\text { a } 45 \text { días }\end{array}$ & \\
\hline $\begin{array}{l}\mathrm{B}= \\
\text { modalidad }\end{array}$ & $\begin{array}{l}\text { b1 }=\text { Pan de tierra } \\
\text { b2 }=\text { Raíz desnuda } \\
\text { b3 }=\text { Deshojado } \\
\text { b4 }=\text { Pseudoestaca }\end{array}$ & $\begin{array}{l}93.46 \\
59.46 \\
46.13 \\
36.29 \\
\end{array}$ & $\begin{array}{r}113.16 \\
64.7 \\
62.1 \\
37.58 \\
\end{array}$ & \begin{tabular}{|c|}
171.32 \\
55.07 \\
70.08 \\
55.26 \\
\end{tabular} & $\begin{array}{c}139.92 \\
63.15 \\
61.46 \\
44.23 \\
\end{array}$ & $\begin{array}{l}517.86 \\
242.38 \\
239.77 \\
173.36\end{array}$ \\
\hline & Totales & 235.34 & 277.54 & 351.73 & 308.76 & 1173.37 \\
\hline
\end{tabular}

\section{DISCUSION}

\section{Porcentaje de prendimiento:}

En lo que respecta a esta variable consideramos que sólo existe diferencia significativa en el factor B, es decir modalidad de trasplante (cuadro 1), al realizar la prueba de Duncan (cuadro 2) notamos hasta dos grupos homogéneos, de los cuales la modalidad con pan de tierra resultó muy superior a los demás, obeteniendo un porcentaje de 86.76 en promedio y el segundo lugar la modalidad de deshojado con un porcentaje de 73.16 en promedio; ambos forman un solo grupo homogéneo.

Por cierto era lógico esperar estos resultados por que la plántula con pan de tierra sufre menos el inpacto del trasplante que bajo las otras formas. Por otro lado el factor A es decir, el momento óptimo de trasplante resultó no significativo, pues los cuatro niveles para este factor presentan casi los mismos promedios.

A pesar de no haber salido significativo la interacción $A$ x $b$, se efectuó el gráfico de los efectos principales, donde notamos (gráfico 1), que la modalidad con pan de tierra sobresale de todas las demás; el gráfico muestra tambien alguna interacción entre los efectos principales que resulta significativo al ANVA, y esto debido a la falta de paralelismo de las líneas 
trazadas, conviene hacer notar que dicho paralelismo se da a 30 y 45 días, inclusive de 15 a 30 días. Finalmente "parece" ser que el mejor momento para trasplantar las plántulas de camu camu es a 30 días después del repique, para verificar esta hipótesis, se procedió a efectuar el análisis de la variable altura de plantas y poder así tener mayores elementos de juicio.

\section{Altura de Plantas:}

El análisis de la variable porcentaje de prendimiento, si bien es cierto nos dio ciertas luces para determinar el momento óptimo y la mejor modalidad, no fue suficiente debido a que efectuando el trasplante muchas plántulas permanecían muy pequeñas y otras tenían un desarrollo excelente en todos los tratamientos; en ese sentido se procedió a realizar el análisis de esta variable. Así el cuadro 5 nos muestra el análisis de variancia de esta variable en donde notamos diferencia significativa en factor A (momento optimo) y en modalidad de trasplante (factor $B)$, así mismo resulto también significativo la interacción $A$ x $B$.

Para el caso del factor $A$, se observa hasta dos grupos homogéneos (cuadro 6 ), de los cuales el nivel A2 es decir a 30 días resulto con el mejor promedio (29.30), muy seguido del nivel A3 (45 dias) con promedio de 25.72 y finalmente el nivel A1 con promedio de 25.12, todas formando un grupo homogéneo.

Para el caso del factor $B$ coincide con los resultados de la primera variable. Así el nivel B1 resulto ser el mejor con 43.13 $\mathrm{cm}$ en promedio y significativo al resto de tratamientos, resultando con el más bajo promedio, el pseudoestaca con 14.44 $\mathrm{cm}$ en promedio.

Estos resultados, no hace más que confirmar, lo reportado por otros autores como Calzada (1985), en el sentido de efectuar el trasplante con pan de tierra y despues de un tiempo prudencial del repique; sin embargo este resultado difiere mucho de lo reportado por Gutierrez (1969), el cual indica que el camu camu debe ser trasplantado a los 9 meses y con una altura de $40 \mathrm{~cm}$. De igual forma difiere mucho lo reportado por Pinedo (1992), el cual recomienda efectuar el trasplante entre los 5 y 9 meses y en la modalidad de raíz desnuda, en ambos casos el periodo de trasplante se alarga entre 5 y 9 meses para llevarlo a terreno definitivo pudiendo llevarlo a 30 días después 
del repique, lo que nos ahorraría entre dos y seis meses de espera.

\section{Interacción A x B :}

Para la variable altura de plántulas, la interacción $A \times B$ resultó significativa, más no así para la variable porcentaje de prendimiento. Así está interacción se encuentra plasmada en el gráfico 2, donde se observa con nitidez que la modalidad con pan de tierra resultó ser el mejor y mucho mayor aún a los 30 días, el resto de modalidades permaneció muy por debajo de la modalidad con pan de tierra.

Asi mismo el gráfico 2 nos muestra también una clara interacción y esto debido a la falta de paralelismo de las líneas trazadas, además hay una deferencia notable entre los efectos principales de los cuatro niveles de A.Finalmente el mismo gráfico nos indica que las interacciones se dan para los niveles de raíz desnuda, deshojado y pseudoestaca, lo cual indudablemente tienen poca importancia para el presente trabajo.

\section{CONCLUSIONES}

El presente porcentaje de prendimiento, sólo el factor B (modalidad trasplante), resultó significativo y dentro de este nivel B1 con pan de tierra, el mejor promedio de prendimiento.

En altura al cuarto mes, resultaron significativos el factor $A$, el factor B y la Interacción A x B.

Que el mejor momento de "llevar" al camu-camu a terreno definitivo corresponde a 30 días después del repique, bajo la modalidad de pan de tierra. 


\section{BIBLIOGRAFIA}

CALZADA, B. J osé, 1985. Ponencia presentada sobre la importancia del camu-camu. 2 da. reunión de Investigación en frutales nativos Iquitos.

GUTIERREZ, R. 1969 Especies Frutales Nativas de la Selva del Perú. Estudio Botánico y de propagación por semilla. Tesis de Ing. Agr. U.N.A. La Molina Lima Perú.

PETER'S CH. VASQUEZ, M. 1987. "Estudios Ecológicos del camu camu. Producción de frutos en poblaciones naturales." Folia Amazónica. I Iquitos: IIAP

PINEDO, M. TANCHIVA E. 1992. Metodología de trasplante a campo definitivo del araza (Eugenia stipitata), camu-camu (Myrciaria dubia) y pijuayo (Bactris gassipaes). Programa de investigación en cultivos tropicales. Iquitos (Perú.)

ROCA N.A. 1965. Estudio químico - bromatológico de Myrciaria paraensis Berg, tesis de química Universidad Mayor de San Marcos. Lima (Perú.)

VASQUEZ M.A. 1991. Estudio Bio-ecológico del camu-camu, ponencia al primer congreso de Ecología. 


\section{Cuadro 10.}

\section{Promedio de altura y otras variables al momento de cada trasplante}

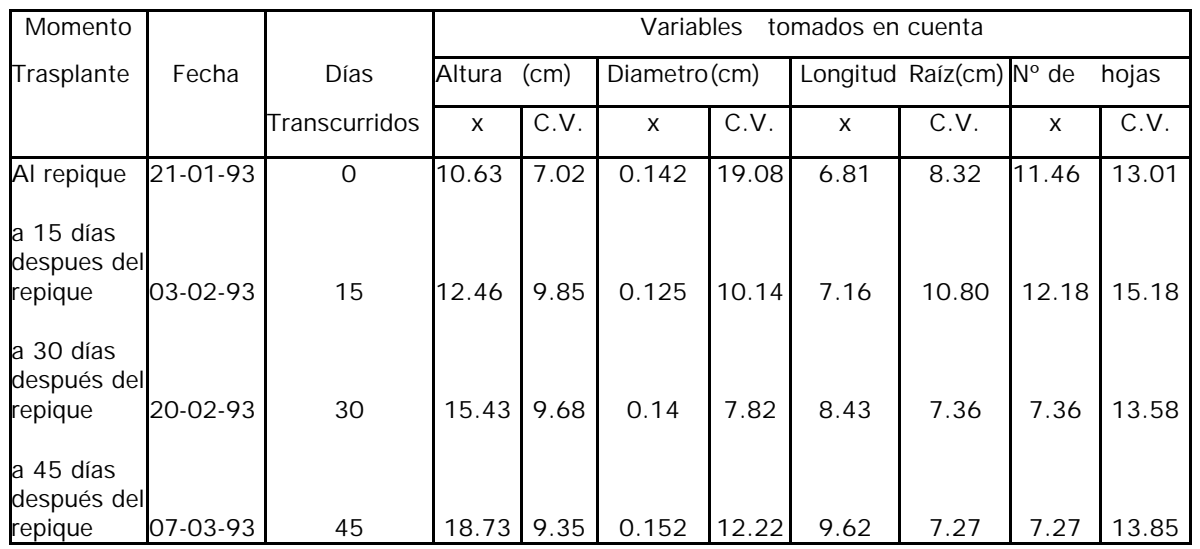


ANEXOS

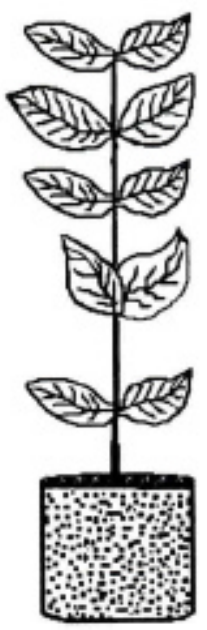

C. TRASPLANTE DESHOJADO

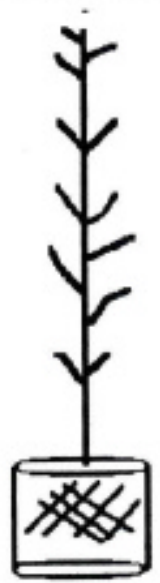

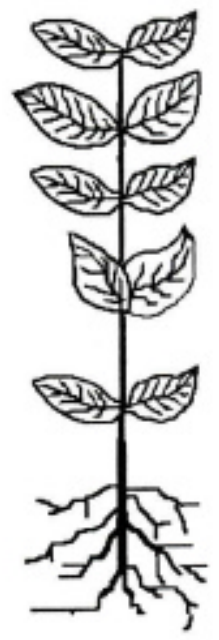

D. TRASPLANTE PSEUdOESTACA

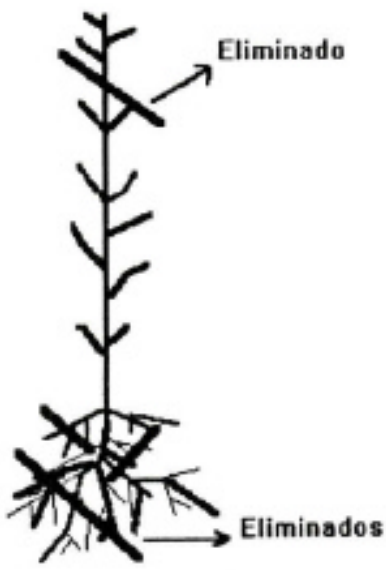

